\title{
Malignant Solitary Fibrous Tumor of the Scalp
}

\author{
Brett M. Shirley • David R. Kang • Aya Hamao Sakamoto
}

Received: 8 July 2014/Accepted: 16 February 2015/Published online: 3 March 2015

(c) The Association of Oral and Maxillofacial Surgeons of India 2015

\begin{abstract}
Solitary fibrous tumors are an uncommon slow growing benign neoplasm originally described as a pleural neoplasm but can also be found in the lung, mediastinum, peritoneum, or any other sites including the head and neck. Malignant solitary fibrous tumors (MSFT) are extremely rare and only few cases have been published in the literature. There have been 19 cases reported of MSFT in the head and neck, but there are no reports of MSFT located within the scalp in the English language literature. We present a case of MSFT arising in the scalp and describe our experience with the clinical presentation, surgical management, and outcome in this pathological condition.
\end{abstract}

\section{B. M. Shirley $(\square)$}

Oral and Maxillofacial Surgery Division, Department of Surgery, Parkland Memorial Hospital, University of Texas Southwestern Medical Center at Dallas, Dallas, TX, USA e-mail: brettshirleyddsmd@gmail.com

\section{B. M. Shirley}

Oral and Maxillofacial Surgery Department, John Peter Smith Hospital, 1500 S Main, Fort Worth, TX 76104, USA

\section{R. Kang}

Department of Oral and Maxillofacial Surgery, Texas A\&M University Baylor College of Dentistry, Dallas, TX, USA

e-mail: david.kang@baylorhealth.edu

\section{R. Kang}

Department of Surgery, Division of Surgical Oncology, Baylor University Medical Center, 3302 Gaston Ave, Dallas, TX 75246, USA

\section{A. H. Sakamoto}

Oral and Maxillofacial Surgery Division, Department of Surgery, University of Texas Southwestern Medical Center at Dallas, 5323 Harry Hines Blvd, Dallas, TX 75390-9109, USA e-mail: aya.hamao-sakamoto@utsoutwetern.edu
Keywords Solitary fibrous tumor $\cdot$ Malignant solitary fibrous tumor - Pleural tumor - Scalp tumor - Head and neck oncology $\cdot$ Neoplasms of the head and neck

\section{Case Report}

A 37 year old female presented to the Oral and Maxillofacial Surgery clinic complaining of a bump on her posterior scalp. She was referred to our clinic by general surgery for evaluation and removal of this lesion. She states that the lesion had slowly begun to increase in size approximately 2 years ago, but was first noticed approximately 4 years ago. She stated that the lesion was very uncomfortable for her and hurt at times when sleeping. On clinical examination, there was a well-circumscribed mass at the right occipital quadrant. The lesion was approximately $5 \times 6 \mathrm{~cm}$ in size. It was soft, not fluctuant, with a sessile base and mildly tender to palpation. No overlying erythema or ulceration was noted.

It was difficult to ascertain the mobility of the lesion due to the location in the posterior occipital region and the thickness of the scalp tissue but appeared to be mobile. No neurological deficits were noted. A Computed Tomography (CT) scan was performed, revealing a well circumscribed $65 \mathrm{~mm} \times 30 \mathrm{~mm} \times 60 \mathrm{~mm}$ right parietal soft tissue mass of heterogeneous densities which was contiguous with the calvarium over $50 \mathrm{~mm}$ without bony erosion or remodeling and sharply defined from adjacent soft tissues (Fig. 1). After the risks, benefits, and alternatives were discussed with the patient, informed consent of excisional biopsy was signed. The patient was then prepped and draped in the standard sterile fashion. An incision was made using a \#15 blade taking an elliptical portion of tissue overlying the lesion. After initial dissection, it was noted that the lesion 


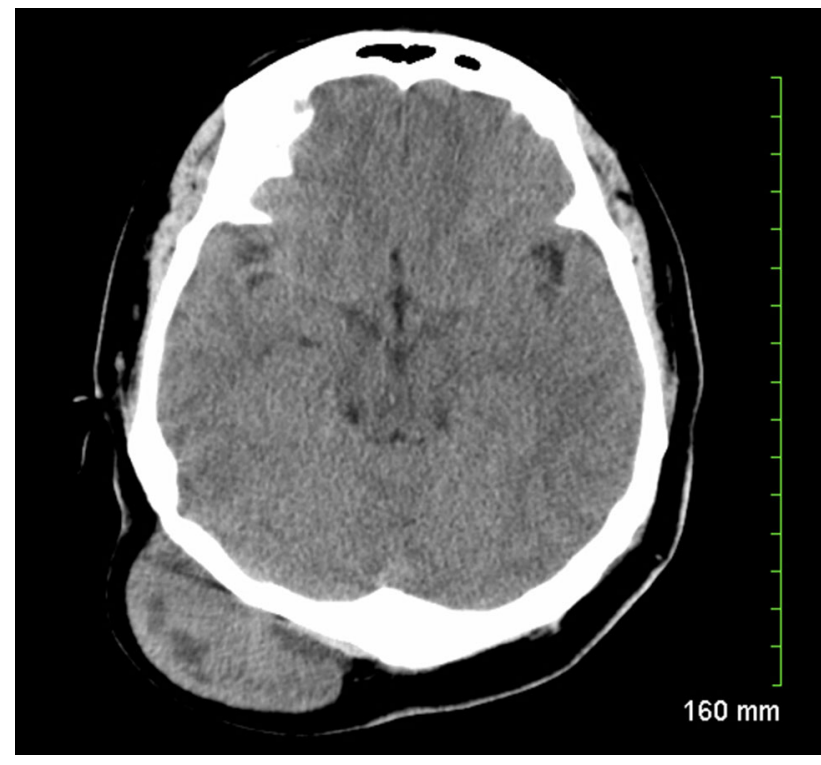

Fig. 1 Axial CT scan without contrast of lesion

was well circumscribed, soft, with areas of fluctuance along the specimen. The lesion was densely adhered to the pericranium and occipital muscles. It was dissected free from these structures taking an additional uninvolved anatomical layer with the specimen. The lesion was excised and found to be well circumscribed, all margins appeared to have been intact (Fig. 2). The site was irrigated copiously with normal saline. The wound was then closed in layers and the specimen sent to pathology.

On microscopic examination, the tumor measured $6.0 \times 4.5 \times 3.5 \mathrm{~cm}$ in size with the tumor abutting the margin. The tumor was hypercellular and composed of spindled cells in a fibrous background (Fig. 3a). There were "stag horn" vessels and peripheral areas of

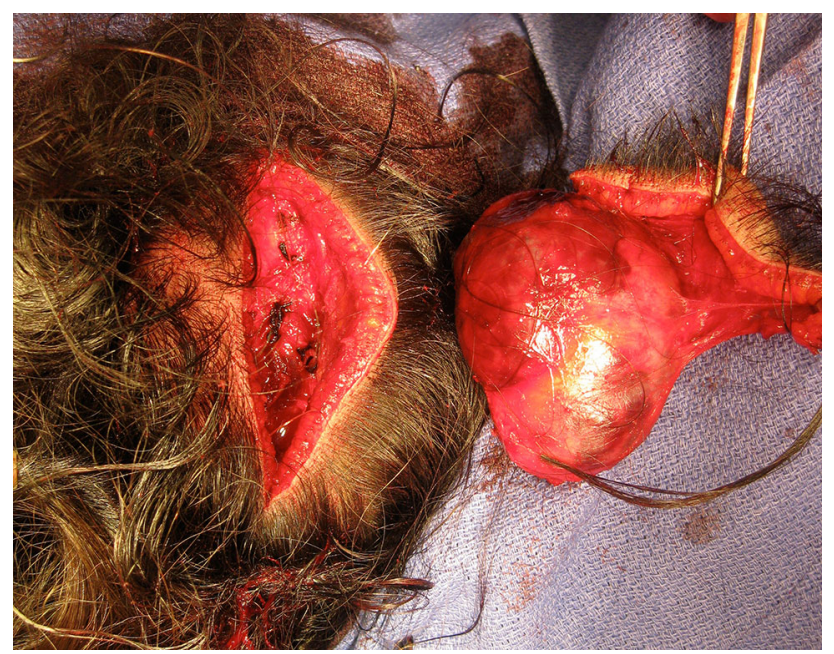

Fig. 2 Intact specimen after scalp excision hemorrhage (Fig. 3b). The tumor cells had prominent nucleoli with rare nuclear pseudoinclusions (Fig. 3c) and moderate nuclear pleomorphism (Fig. 3d). A mitotic count was performed with 4 mitoses/10HPF. Immunohistochemical stains were performed including FLI-1, BCL-2, CD99, CD34, CD57, EMA, HMB-45, S100, and CD 31. The tumor cells were diffusely reactive for FLI-1, BCL-2 (Fig. 4), CD99, and CD 34. The CD57, EMA, HMB-45, S100, and CD 31 stains were negative within the cells of interest. The differential diagnosis included synovial sarcoma and primitive neuroectodermal tumor (PNET), therefore, FISH assays for SYT $(\mathrm{X} ; 18)(\mathrm{p} 11.2 ; \mathrm{q} 11.2)$, and EWSR1 $(11 ; 22)(\mathrm{q} 24 ; \mathrm{q} 12)$ translocations were sent and negative, therefore ruling out synovial sarcoma [SYT $(\mathrm{X} ; 18)$ translocation] and PNET [EWSR1 $(11 ; 22)$ translocation]. Therefore, the histological diagnosis of malignant solitary fibrous tumor was given. The tumor was found to be abutting the margin, therefore, the patient was taken to the operating room for a wedge resection with the new margin $1 \mathrm{~cm}$ from the initial site. All margins taken were negative for residual tumor. Moreover, no clinical evidence or recurrence, or distant metastasis was observed for 18 months.

\section{Discussion}

SFT in the head and neck was first described by Witkin when they reviewed a series of six cases in 1991 but was originally described as a pleural tumor [1-4].

SFT most often involves the visceral pleura and is attached to the serosal surface [5]. The etiology of these tumors were unclear and was originally thought to originate from mesothelial cells, but Hanau et al. have demonstrated features of undifferentiated mesenchymal cells without evidence of mesothelial differentiation [6]. There is also controversy with hemangiopericytoma and SFTs and many feel that tumors previously classified as hemangiopericytoma are in fact SFTs [7]. SFTs have been described in many different sites besides the serosal surfaces of the lungs including mediastinum, peritoneum, liver, nasal cavity, tongue, and orbit but all show the same characteristics as the SFT of the serosal surfaces $[1,2,8-$ 10]. MSFTs are rare but may arise de novo or from an existing SFT [10]. SFTs are rarely found in the head and neck region and account for approximately $16 \%$ of all cases [11]. Cox et al. [2] reviewed the English language literature and found 153 cases of SFT reported with 10 of these cases displaying malignancy. These cases of MSFT were located in the buccal mucosa, upper eyelid, palatine tonsil, frontoethmoid sinus, nasal cavity, cheek, sphenoid sinus, tongue, floor of mouth, and orbit. The sex was split equally with 5 females and 5 males with an average age of 
Fig. 3 a H\&E stain low power $(\times 10)$ showing hypercellular spindle cells. b H\&E stain medium power $(\times 40)$ staghorn vasculature. $\mathbf{c} \mathrm{H} \& \mathrm{E}$ stain high power $(\times 60)$ showing nuclear pseudoinclusion and mitosis 4/10 HPF. d H\&E stain high power $(\times 60)$ nuclear pleomorphism
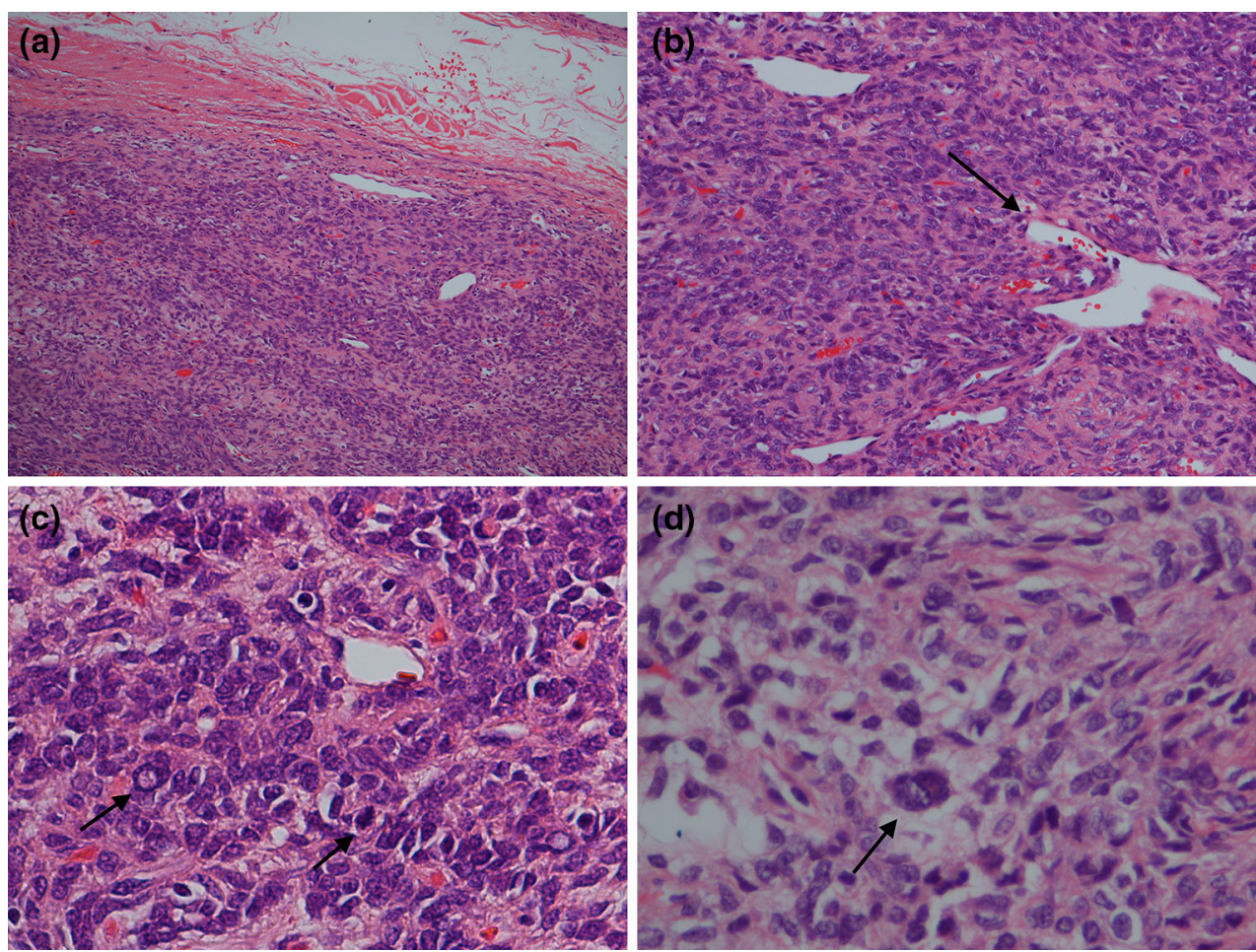

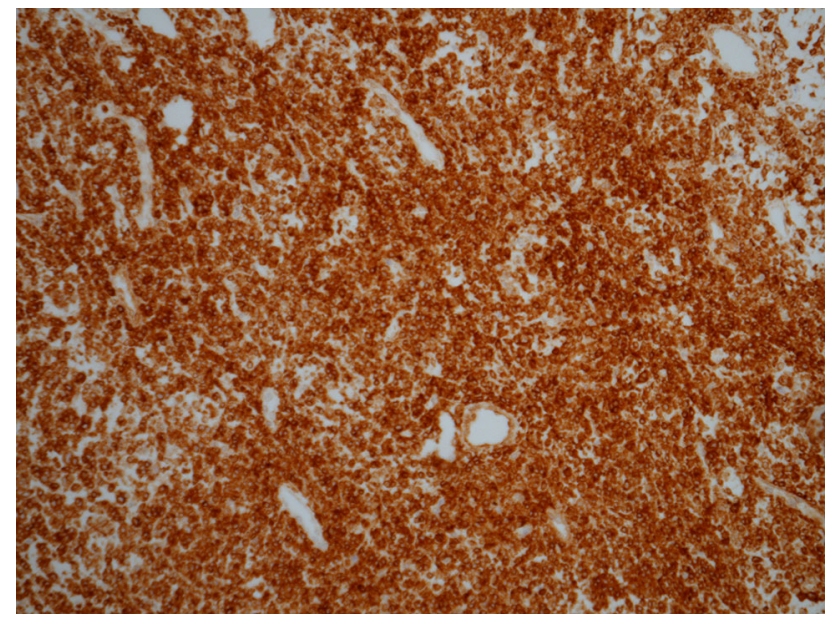

Fig. 4 Bcl-2 stain strongly positive

57 ranging from 27 to 78 years old. Yang et al. reported another additional 24 cases of SFT with 9 of those cases reported as being malignant. In their series of 9 patients with MSFT, three were identified in the tongue, two in the infratemporal fossa, one in the parotid, one in the mandible, one in the temporomandibular joint, and one in the pterygopalatine fossa. The sex was also split equally with 4 females and 5 males with an average age of 43 ranging from 25 to 77 years [12].

The diagnosis of SFT is based on the branching pattern of small and large vessels, a "staghorn" configuration with bland, round to fusiform cells arranged around elaborate vasculature. The cells have round to oval nuclei and may be spindled. SFT cells stain for CD34, and CD99 and variably stain for epithelial membrane antigen, bcl-2, and smooth muscle actin and limited reactivity to S-100 [13]. The diagnosis of MSFT versus SFT is based on mitotic activity ( 4 mitoses per 10 high powered field), $>5 \mathrm{~cm}$ size of lesion, high degree of cellularity, presence of immature and pleomorphic tumor cells and foci of hemorrhage and necrosis [14].

The differential diagnosis for MSFT will include SFT, synovial sarcoma, nerve sheathe tumors, fat and smooth muscle tumors [1]. Chiu et al. retrospectively reviewed 398 Taiwanese patients with scalp tumors and found that basal cell carcinoma (41.2\%) and squamous cell carcinoma $(16.6 \%)$ were the most frequent histological type of scalp malignancy. Tumors arising from the epidermis made up $57.8 \%$ of scalp malignancies. Metastatic tumors (12.8\%) were third in their series with recognized primary tumors originating from lung, colon and breast cancers. Other less common tumors include tumors of the skin appendages differentiated from follicular (2.3\%), sebaceous (4.3), or eccrine $(3.5 \%)$, and tumors of the soft tissue including angiosarcoma $(6.8 \%)$, dermatofibrosarcoma protuberans $(2.8 \%)$, and leimyosarcoma (2.0\%) [15].

Surgical resection is the treatment of choice and with adjuvant treatment of chemotherapy and radiation therapy if there are positive margins or recurrences [16]. MSFT 
appears to be radiation-responsive with fifty gray or higher reducing local recurrence [16]. In the series of 9 patients with MSFT, Yang et al. found the locoregional recurrent rate of MSFT to be $11 \%$ with a rate of metastasis of $33 \%$. It was also noted that the number of mitoses per high power field correlated with a poor prognosis and found that patients who died from the disease had on average $41.3 \mathrm{mi}-$ toses versus those with 6.2 mitoses per high power field who were disease free [12]. In contrast, Cox et al. [2] had not found cases with malignant microscopic features to behave more aggressively than their benign counterparts. They believe complete resection with negative margins to be curative without need for additional treatment, however, they did not note the number of mitoses per high power field, therefore, it is unclear how aggressive the MSFTs were in their review.

MSFTs are distinguished from benign SFTs histologically with the number of mitoses per high power field, $>5 \mathrm{~cm}$ size, high degree of cellularity, presence of immature and pleomorphic tumor cells and foci of hemorrhage and necrosis. The number of mitoses per high power field appears to be correlated with prognosis. Fewer than a 2 per 10 high power field appears to indicate a favorable prognosis and 4 or more per 10 high power field correlates with recurrent disease and metastasis [12]. Solitary fibrous tumors of the head and neck are rare and malignant solitary fibrous tumors of the head and neck are even more uncommon. This is the first reported case of MSFT of the scalp, and MSFT should be included in the differential diagnosis of head and neck lesions.

\section{References}

1. Bishop JA, Rekhtman N, Chun J et al (2010) Malignant solitary fibrous tumor: cytopathologic findings and differential diagnosis. Cancer Cytopathol 118:83
2. Cox DP, Daniels T, Jordan RK (2010) Solitary fibrous tumors of the head and neck. Oral Surg Oral Med Oral Pathol Oral Radiol Endodontol 110:79

3. Witkin GB, Rosai J (1991) Solitary fibrous tumor of the upper respiratory tract. A report of six cases. Am J Surg Pathol 15:842

4. England DM, Hochholzer L, McCarthy MJ (1989) Localized benign and malignant fibrous tumor of the pleura: a clinicopathalogic review of 223 cases. Am J Surg Pathol 13:640

5. Hanau CA, Mietinen M (1995) Solitary fibrous tumor: histological and immuno-histochemical spectrum of benign and malignant variants presenting at different sites. Hum Pathol 26:440

6. Fletcher CD (2006) The evolving classification of soft tissue tumors: an update based on the new WHO classification. Histopathology $48: 3$

7. Migita M, Yoshino M, Kobayashi D et al (2012) A large solitary fibrous tumor of the tongue. J Oral Maxillofac Surg 70:871

8. Chan JK (1997) Solitary fibrous tumor-everywhere, and a diagnosis in vogue. Histopathology 31:568

9. Vafiadou M, Dimitrakopoulos I, Georgitzikis I et al (2008) Solitary fibrous tumor of the tongue: case report and literature review. Int J Oral Maxillofac Surg 37:1067

10. Yokoi T, Tsuzuki T, Yatabe Y et al (1998) Solitary fibrous tumor: significance of p53 and CD34 immunoreactivity in its malignant transformation. Histopathology 32:423

11. Yang XJ, Zheng JW, Ye WM et al (2009) Malignant solitary fibrous tumors of the head and neck: a clinicopathological study of nine consecutive patients. Oral Oncol 45:678

12. Fletcher CD, Unni KK, Mertens F (2002) World Health Organization classification of tumors. Pathology and genetics of tumors of soft tissue and bone. Springer, New York, pp 86-88

13. Enzinger FM, Smith BH (1976) Hemangiopericytoma: an analysis of 106 cases. Hum Pathol 7:61

14. Chiu C, Lin C, Kuo T, Kuan Y et al (2007) Malignant cutaneous tumors of the scalp: a study of demographic characteristics and histologic distributions of 398 Taiwanese patients. J Am Acad Dermatol 56:448

15. Wong PP, Yagoda A (1978) Chemotherapy of malignant hemangiopericytoma. Cancer 41:1256

16. Pisters PW, Harrison LB, Woodruff JM et al (1994) A prospective randomized trial of adjuvant brachytherapy in the management of low-grade soft tissue sarcomas of the extremity and superficial trunk. J Clin Oncol 12:1150 